\title{
A Modified Tie Technique for Securing Endotracheal Tubes
}

\author{
Huw RF Walters MB BChir, Holly E Young, and Peter J Young MD FFICM FRCA MBChB
}

\begin{abstract}
BACKGROUND: Unintentional extubation is a well-documented and potentially life-threatening phenomenon. There is little research into the factors that lead to unintentional extubation, and therefore how to prevent it. Endotracheal tubes (ETTs) are commonly secured with $1 \mathrm{~cm}$ twill tape, but there is little evidence on how to best tie them in place. We have devised a new knot for securing these tubes in place, and we have assessed its degree of slippage when exposed to both perpendicular and sliding forces in comparison with other commonly used knots. METHODS: We constructed a weighted apparatus to test the effects of these forces and measure whether conventional ETTs slipped. We tested the knots both wet and dry to better simulate the clinical environment. We also conducted a manual manipulation test in which a single operator attempted to slide the knot along the ETT. Each knot was tested 10 times for the weight test under both wet and dry conditions, and 8 times for the slip test. RESULTS: The normal knot slipped when weighted both wet and dry, while the clove hitch failed the weight test only when wet. Both the modified cow hitch and double hitch withstood all weights. Only the double hitch did not slip under any circumstances on either test. CONCLUSIONS: The new double hitch was superior to all conventional knots tested. It did not slip under any conditions and therefore may be of use to prevent unintentional extubation, particularly in the ICU setting. Key words: unintentional extubation; tie; twill tape; knot; double hitch; endotracheal tube. [Respir Care 2018;63(4):424-429. (C) 2018 Daedalus Enterprises]
\end{abstract}

\section{Introduction}

Endotracheal tubes (ETTs) are commonly used to secure airways of critically ill, or anesthetized patients. They are subject to a number of external forces and slippage, and it is well documented that these tubes are susceptible to coming out accidentally. ${ }^{1}$ Unintentional extubation can be a life-threatening event, ${ }^{2}$ so it is essential that ETTs are secured in position, especially in the context of the critically ill, where patients are commonly intubated for prolonged periods of time. ${ }^{3}$ In clinical practice, there is a wide variability of methods used to secure the ETT including

Dr Walters and Ms Young are affiliated with the School of Clinical Medicine, Cambridge University, United Kingdom. Dr Young is affiliated with The Queen Elizabeth Hospital NHS Foundation Trust, Gayton Road, Kings Lynn, Norfolk, United Kingdom.

The authors have disclosed no conflicts of interest.

Correspondence: Huw RF Walters MB BChir, School of Clinical Medicine, Cambridge University, Cambridge, UK. E-mail: Huw.Walters@nhs.net.

DOI: $10.4187 /$ respcare.05655 using adhesive tape, cloth tape ties, and commercial ETT holders. We chose to study an improvement in the common methods used to tie ETTs.

While unintentional extubation is well documented, there is little available evidence regarding the best way to secure ETTs, and the literature that does exist is inconclusive. ${ }^{4}$ Twill tape is commonly used to secure these tubes, but there is little evidence as to which knot provides maximum security. ${ }^{5,6}$ The Royal College of Anesthetists offers no formal guidance on the best way to secure these tubes, stating that "the precise method of securing an ETT will depend on the nature of the operation and the preference of the anesthetist. What is important is that the tube is secure but is not impeding the surgery" (personal communication, 2016).

Our study compares the usefulness of 3 commonly used knots for securing ETTs: the simple or normal knot, the clove hitch knot, and the modified cow hitch knot. We also tested a novel secondary anchoring technique that we called the double hitch. These knots were tested to assess whether the ETTs slipped out of position, first against incrementally increasing outward pull forces with taught lateral fixation tape, and then using finger pressure with slack lateral fixation. 


\section{Modified TIE Technique for ETTs}

\section{Methods}

We performed an Internet literature and video search to identify common methods for tying ETTs in critical care patients. An artificial apparatus was created to simulate a secured ETT being subjected to outward pull forces meant to mimic unintentional extubation. This consisted of an ETT (Portex, Hythe, United Kingdom) with an inner diameter of $8.0 \mathrm{~mm}$ secured with one of the knots at the $25-\mathrm{cm}$ mark, suspended between 2 surfaces with a gap of $5 \mathrm{~cm}$ on either side. Standard 1-cm wide synthetic twill tape was used for each knot. The loose ends of tape were secured in such a manner that they could not slip, creating a stable, replicable system for assessing the strength of each knot (Fig. 1). A sealed-envelope technique was used to randomize the knot tied each time.

In the weight test, the slip connector at the ventilator end of the tube was clamped and sequentially loaded against gravity with $0.5 \mathrm{-kg}$ weights (up to a maximum of $5 \mathrm{~kg}$ ), waiting $10 \mathrm{~s}$ between each addition to assess whether any slippage occurred. This was replicated 10 times with each dry knot, and a further 10 times with the knot wetted by submerging in water for $5 \mathrm{~min}$ to simulate the dampness of clinical environment. ${ }^{6}$

In the manual manipulation test, the knots were tightened to a varying degree with the perpendicular pull described above. To simulate more insidious slippage, the experiment was repeated using finger pressure to attempt to slide the knot along the tube.

For both tests, a knot was said to have failed if there was slippage of $>3 \mathrm{~cm}$ from the defined start point. A single individual was responsible for all knot tying, and a second individual performed all testing to standardize conditions.

\section{Knots Tested}

The normal knot (Fig. 2) was tied with 2 left-over-right loops then tightened. The clove hitch (Fig. 3) was tied as per convention: 2 loops made in the tape, passed over the

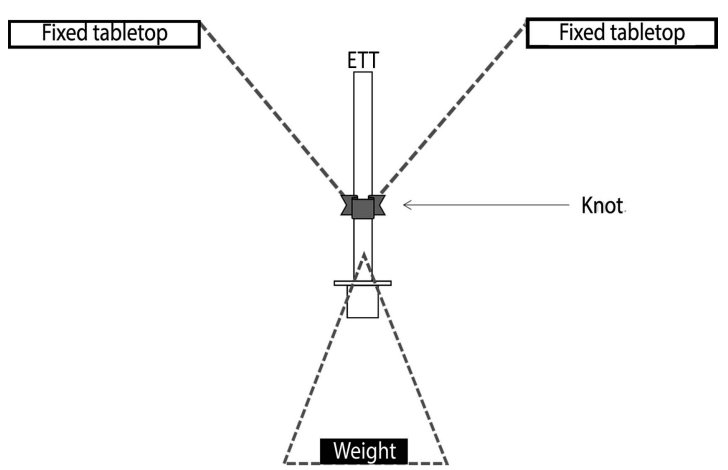

Fig. 1. The apparatus used for the weight test. Weights were sequentially loaded to increase the force on the knot.

\section{QUICK LOOK}

\section{Current knowledge}

There is very little research regarding the most appropriate way to secure endotracheal tubes, despite being subject to a number of external forces. Unintentional extubation is a serious complication of tube slippage.

\section{What this paper contributes to our knowledge}

The type of knot tied onto an endotracheal tube affects the forces that it can withstand before slippage. We have shown, using a weighted model, that some knots were more appropriate for securing endotracheal tubes. The double hitch knot was capable of preventing slippage in our model.

tube and tightened to secure. ${ }^{7}$ The modified cow hitch (Fig. 4) was a conventional cow hitch/lark's head tied by doubling the tape, passing the ends around the tube and through the loop, ${ }^{8}$ and further secured with a normal knot. The double hitch (Fig. 5) was a secondary fixation knot using the free ends of tape after an ETT tie (in this case, the normal knot) as described above. The free ends of the ETT knot tie were passed up and around the slip connector at the end of the tube for 1 complete turn and secured with a normal knot. The slip connector was connected to the ventilator circuit to ensure that it was not impeded in any way by the double hitch knot. The rolling (magnus) hitch, a knot similar to the clove hitch, was not tested because previous studies have shown only marginal differences in effectiveness (both more and less slippage) than the clove hitch. ${ }^{2}$

Study approval was obtained from the institutional review board. The study involved no human tissue or subjects and was deemed to be exempt from ethical approval in line with the Integrated Research Application System.

\section{Statistical Analysis}

The double hitch knot was compared to the 3 conventional knots individually in each state (wet, dry, weight, and slip) using a Fisher's exact test and the Bonferroni correction.

\section{Results}

The double hitch was the only effective knot for the weight $(P<.001)$ and manual manipulation test $(P=.001)$ in both wet and dry conditions. The modified cow hitch was superior to the clove hitch and the normal knot when wet during the weight test, but it was ineffective in the manipulation test. Neither the modified cow hitch nor the double hitch slipped at any force, wet or dry, while the clove hitch 


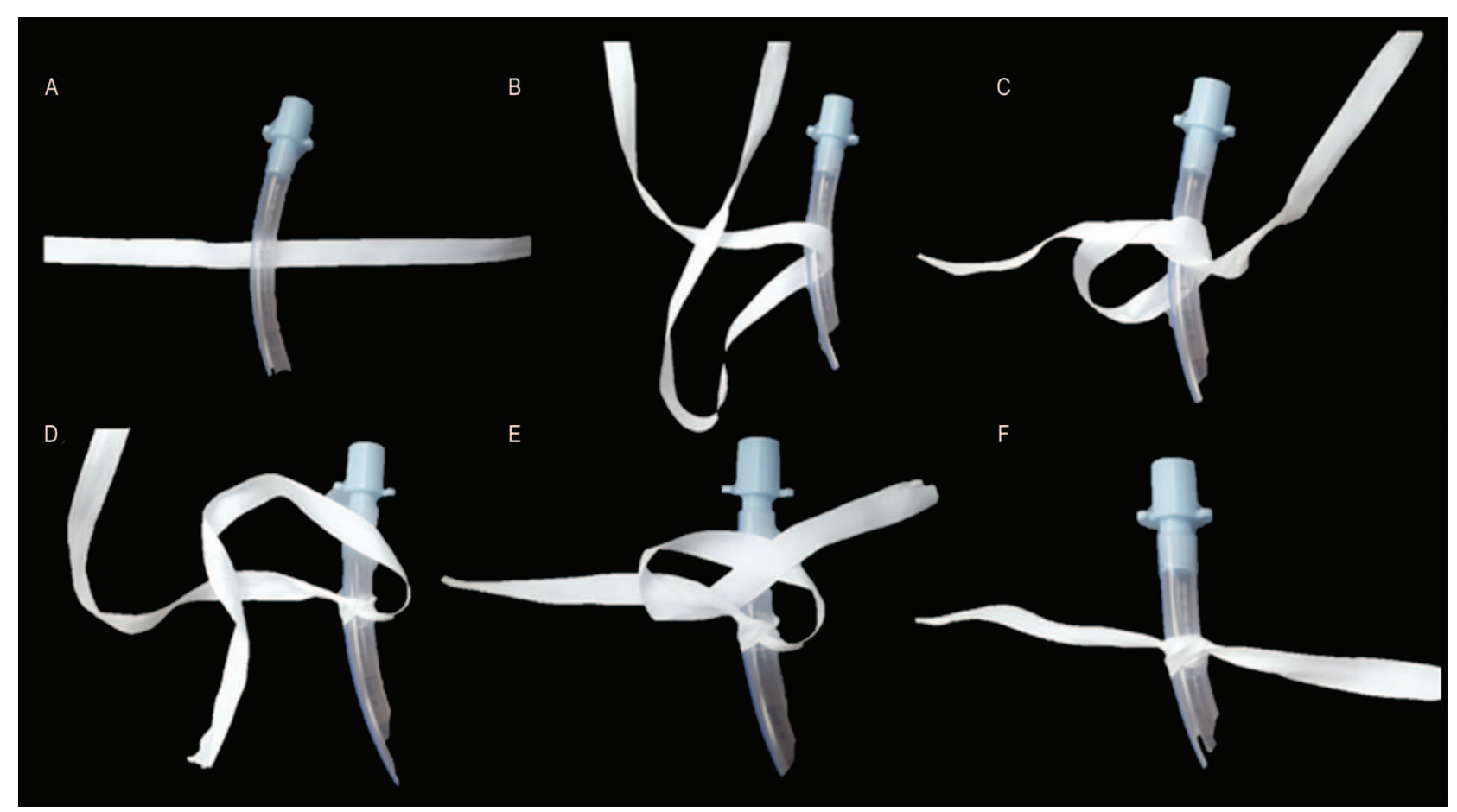

Fig. 2. The normal knot. A: Overlap the 2 ends of tape. B: Pass one end through the loop created. C: Tighten. D, E, and F: Repeat steps A-C again.

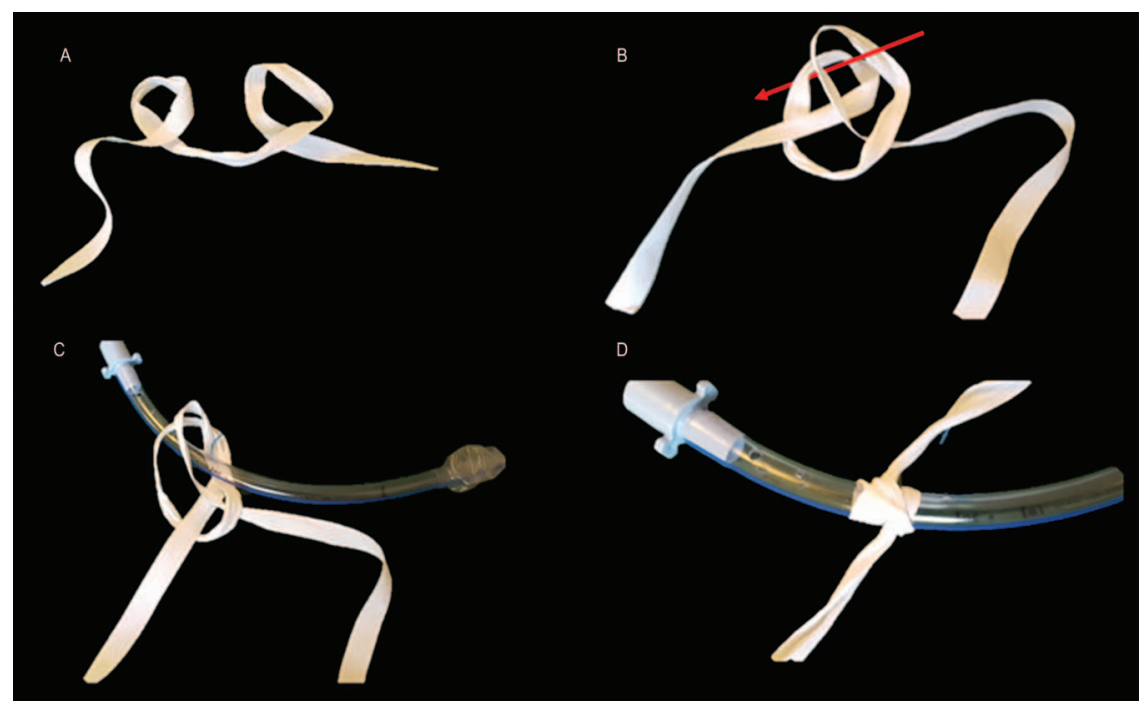

Fig. 3. The clove hitch. A: Form 2 loops in the tape (arrow). B: Overlap the loops and pass over the endotracheal tube. C: Pull tight. D: The completed knot.

slipped when wet (all 10 attempts, range 20-45 N) but not when dry (Fig. 6). The normal knot slipped below $50 \mathrm{~N}$ of force in 2 cases while dry and in all wet cases, with a range of forces from $15-25 \mathrm{~N}$.

While the modified cow hitch did not slip with weight, it was moveable in all manual manipulation test conditions. The only knot that did not slip in the direction of extubation with manual force was the double hitch (Fig. 7).

\section{Discussion}

Unplanned extubation is a common and major iatrogenic complication in the ICU with a reported incidence as high as $2-16 \% .^{2}$ Complications include loss of airway, airway trauma, bronchospasm, difficult re-intubation, cardiopulmonary deterioration, ventilator-associated pneumonia, hypoxemia, and laryngeal and pharyngeal trauma. ${ }^{9}$ 


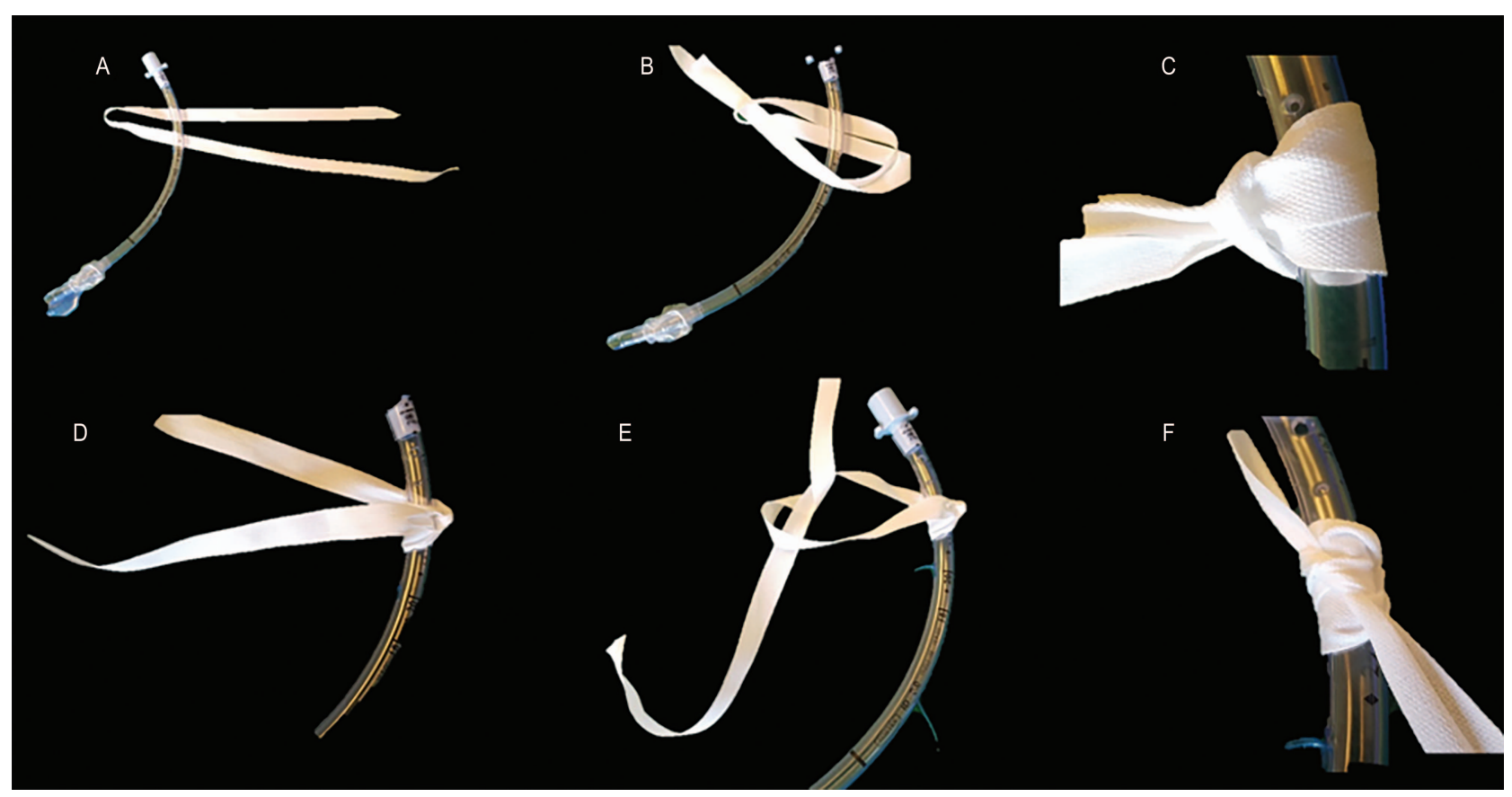

Fig. 4. The modified cow hitch. A: Make a loop and pass under/around the endotracheal tube. B: Pass the loose ends through the loop. C: Pull ends tight. D: Pass one loose end around the tube. E: Tie a normal knot. F: Pull tight.

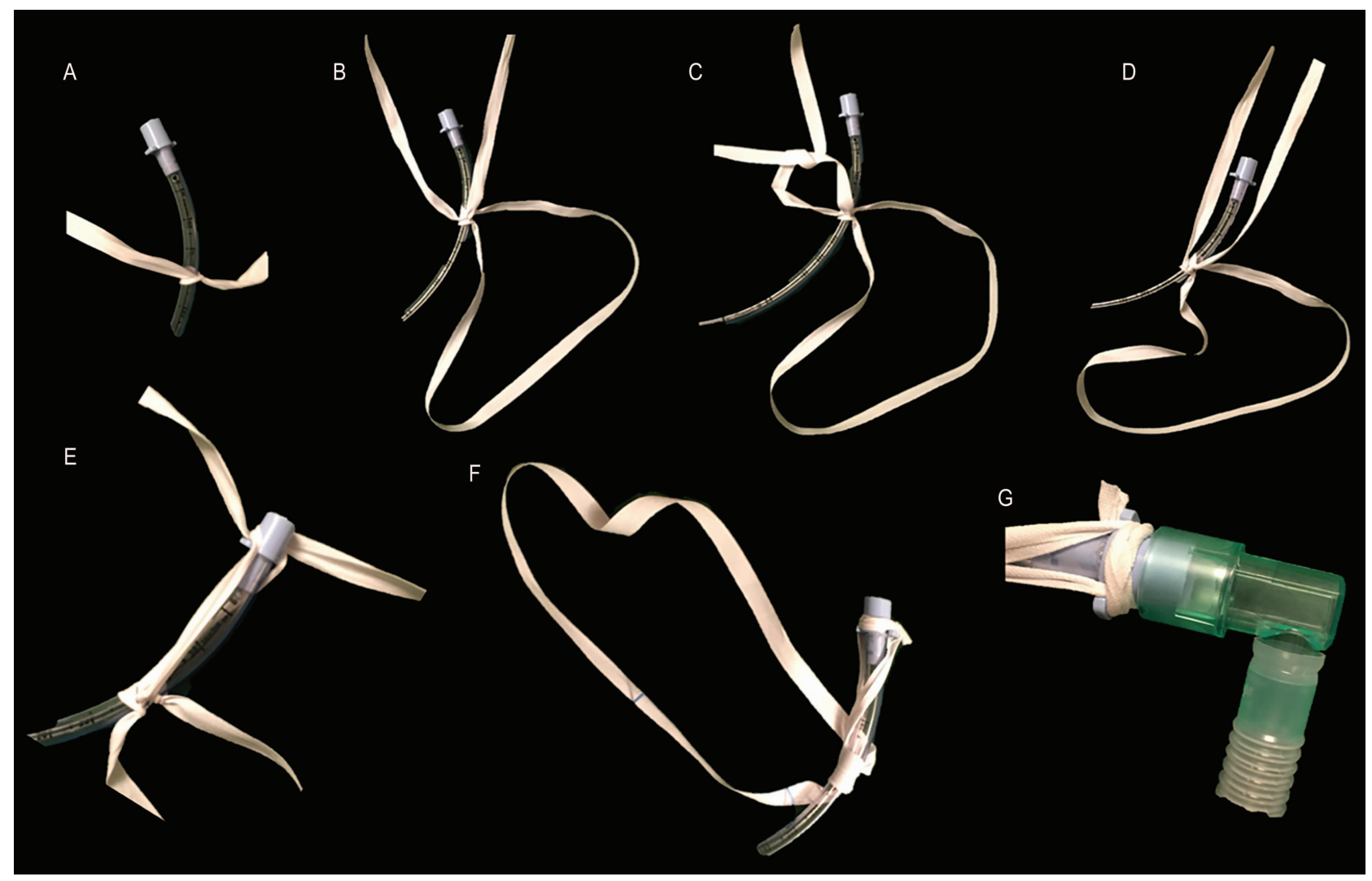

Fig. 5. The double hitch. A: For the first knot, tie a normal knot. B: Tie a second normal knot, forming a loop to secure to the patient. C-D: Join the 2 knots with the third knot. E: Loop free ends around slip connector. F: Tie with a normal knot and trim ends to form the finished knot. G: The knot does not interfere with the connector attachment. 


\section{Modified Tie Technique for ETTs}

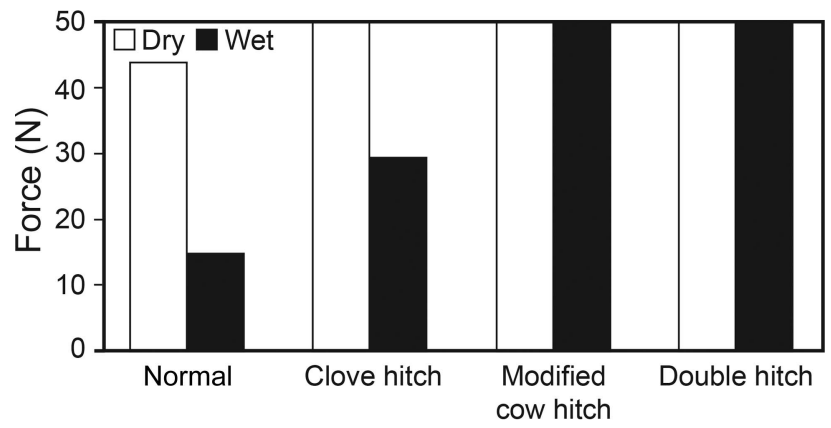

Fig. 6. The mean static force withstood before 3-cm slippage by each knot, under both wet and dry conditions.

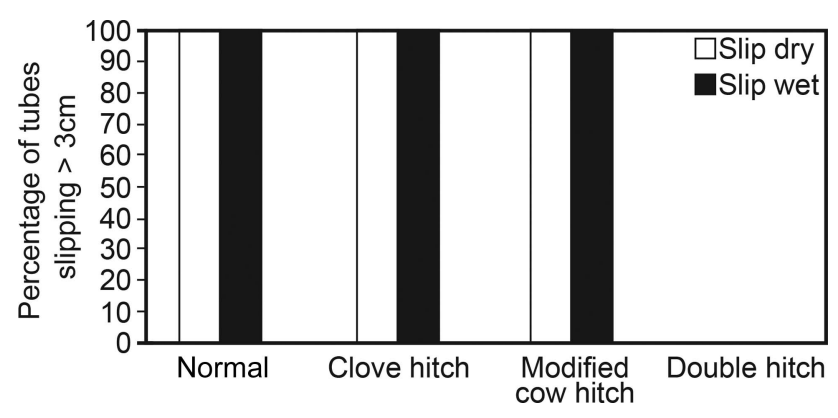

Fig. 7. The percentage of knots slipping $>3 \mathrm{~cm}$ under manual manipulation, performed under both wet and dry conditions. The double hitch did not slip in any instance.

Inadequate sedation has also been identified as increasing the risk of unintentional extubation, presumably in part due to the risk of increased forces on the ETT tie, although there is insufficient research into this area. Nurse work load, procedure standardization of securing the ETT, and the use of hand restraints have been reported to reduce unintentional extubation. ${ }^{1}$ Unintentional extubation may also be reduced by adequate fixation of the tube, and a better secured ETT may also allow for a concurrent reduction of sedation where desirable. ${ }^{10}$

We have shown that different techniques in tying ETTs result in different strengths and different propensities for slippage of the tube. While all 3 knots held well when dry, this does not adequately represent the true clinical setting during longer periods of intubation, especially with knots tied close to the mouth. Conventional knots are able to withstand some force before slippage, but the risk of accidental extubation is present at higher forces. The types of forces applied to the tubes were designed to be similar to what may occur in the clinical setting and represents the propensity of these tubes to slip when wet.

Cloth tape is commonly used to secure ETTs, but there is very little literature on the best way to secure the tube. ${ }^{11,12}$ Many variations can be seen, even within departments, which perhaps represent preferences by health care staff for ease of tying/loosening. It is, however, important to recognize that some of these knots may not be adequate for their purpose and may put patients at risk of unintentional extubation. We have shown that 3 common conventional knots are subject to slippage when external forces are applied to the ETT, which may explain in part the processes that cause some episodes of unintentional extubation. These knots may be suitable for anesthetic usage because of the relatively short duration of intubation in comparison with the critical care setting.

The double hitch knot we have described is designed to prevent tube slippage, and we have shown that it is superior to more conventional knots tested. By virtue of looping over the ventilator slip connector, there are 2 fixation points, tethered against one another, which prevents slippage of the knot on the ETT in the direction that risks extubation. Although the upper portion of the double hitch knot is close to the connecting portion of the ETT, if correctly tied it does not interfere with adequate connection of the ventilator tubing and therefore should have no negative effect on clinical practice. We recommend the adoption of the double hitch for cases of long-term intubation to prevent tube slippage and ensure safety of intubation.

\section{Limitations}

This study is limited in that it was not performed in a clinical setting, and we therefore cannot be sure that we have replicated all variables in play during unintentional extubation. For example, the loop around the neck of the patient can become slack or undone and risk unintentional extubation. Every attempt was made to ensure testing was as similar to real life as possible, although it is difficult to fully and accurately simulate these clinical variables. We ensured optimal tying of each knot, whereas in a clinical setting the knots may be tied by any number of practitioners in many different circumstances, which may result in suboptimal knots on occasion.

An improvement to this study would be to allow the knots to wait for some time to replicate the length of time that these knots are left in situ. Over time it is reasonable to expect these knots to loosen, and they may be more likely to slip. Knots were soaked for only $5 \mathrm{~min}$, which does not reflect the clinical setting, although even this short time increased the slippage dramatically in all the traditional knots tested, so a further extension of time was deemed unnecessary.

In the ICU setting, ETTs are often subject to multiple small forces in many directions over time. These forces are difficult to reliably replicate and therefore were not tested in this situation. However, these small tugging forces may be another stress on the ETT knot and may lead to unintentional extubation at lower forces than shown here. Another limitation is that the weight test produced prolonged forces in the same linear direction, which may not repre- 


\section{Modified TIE Technique for ETTs}

sent all forces encountered clinically, such as multidirectional jerking and twisting motions. Another limitation is that this knot can only be effective on ETTs with protrusions on the slip adapter; these are not present on all types of tube, so the double hitch may not always be appropriate.

\section{Recommendations}

While conventional knots may be suitable for procedures with short intubation times, we have shown that for longer use, these knots have a propensity to slip. The risk of unintentional extubation can be reduced through the use of the double hitch knot reinforcing the primary knot on the ETT, which we have shown is more effective at holding tubes in place.

\section{Conclusion}

We have demonstrated a new knot for securing ETTs. The double hitch is easy to tie and requires minimal training. This knot reinforces conventional knots, providing a greater ability to resist movement. By reducing outward slippage of the ETT, this knot may reduce the incidence of unintentional extubation and therefore improve safety for intubated patients. We have introduced this into clinical practice in our ICU.

\section{REFERENCES}

1. Chevron V, Ménard JF, Richard JC, Girault C, Leroy J, Bonmarchand G. Unplanned extubation: risk factors of development and pre- dictive criteria for reintubation. Crit Care Med 1998;26(6):10491053.

2. Boulain T. Unplanned extubations in the adult intensive care unit: a prospective multicenter study. Association des Réanimateurs du Centre-Ouest. Am J Respir Crit Care Med 1998;157(4 Pt 1):1131-1137.

3. Kapadia FN, Bajan KB, Raje KV. Airway accidents in intubated intensive care unit patients: an epidemiological study. Crit Care Med 2000;28(3):659-664.

4. Gardner A, Hughes D, Cook R, Henson R, Osborne S, Gardner G. Best practice in stabilisation of oral endotracheal tubes: a systematic review. Aust Crit Care 2005;18(4):158, 160-165.

5. Lai M, Inglis GDT, Hose K, Jardine LA, Davies MW. Methods for securing endotracheal tubes in newborn infants. Cochrane Database Syst Rev 2014(7):CD007805.

6. Lovett PB, Flaxman A, Stürmann KM, Bijur P. The insecure airway: a comparison of knots and commercial devices for securing endotracheal tubes. BMC Emerg Med 2006;6:7.

7. Kauffman LH. Knots and Physics. Singapore: World Scientific; 2001.

8. Hage JJ. Heraklas on knots: sixteen surgical nooses and knots from the first century A.D. World J Surg 2008;32(4):648-655.

9. Mort TC. Unplanned tracheal extubation outside the operating room: a quality improvement audit of hemodynamic and tracheal airway complications associated with emergency tracheal reintubation. Anesth Analg 1998;86(6):1171-1176.

10. Kiekkas P, Aretha D, Panteli E, Baltopoulos GI, Filos KS. Unplanned extubation in critically ill adults: clinical review. Nurs Crit Care 2013;18(3):123-134.

11. Farbod F, Tuli P, Robertson BF, Jackson IT. Endotracheal tube fixation methods for optimal stability: a comparison of adhesive tape, suture, and tape-suture fixation. J Craniofac Surg 2010;21(4): 1250-1251.

12. Shimizu T, Mizutani T, Yamashita S, Hagiya K, Tanaka M. Endotracheal tube extubation force: adhesive tape versus endotracheal tube holder. Respir Care 2011;56(11):1825-1829. 